\title{
Chiral symmetry and mixing of axial and vector correlators in matter
}

\author{
Boris Krippa \\ Institute for Nuclear Research of the Russian Academy of Sciences, Moscow Region 117312, \\ Russia.
}

\begin{abstract}
The effect of mixing of the vector and axial vector correlation functions in the nuclear medium arising from the interaction of nuclear pions with corresponding interpolating currents is considered. It is shown that the mass difference between $\rho$ and $a_{1}$ meson gets smaller with the increase of the nuclear density reflecting the phenomena of partial restoration of chiral symmetry whereas the absolute values of meson masses may both decrease and increase in nuclear medium depending on the model used for the phenomenological spectral density.
\end{abstract}

PACS: 11.30.Rd, 11.55.Hx, 21.65.+f

During the last few years the behaivior of vector mesons in the dence and/or hot matter attracted much attention. Among them $\rho$-meson is of particular interest as, due to rather significant width, $\rho$-meson decays mostly in nuclear interior and dilepton invariant spectrum should thus carry the direct information on the $\rho$-meson properties in the nuclear medium [1]. From the theoretical point of view the situation is far from clear. According to Brown and Rho [2] the rho-meson mass should decrease in baryon matter like the chiral order parameter (BR-scaling). Indeed, several calculations in the framework of QCD sum rules [3.4], devoted to the study of density dependence of the vector meson masses, suggest significant drop of the $\rho$-meson mass with density (about $20 \%$ at the normal nuclear density). This is the remarkable fact since the decrease of the rho-meson mass in the nuclear matter may indicate the partial restoration of chiral symmetry. In [3] nuclear matter was assumed to be a system of nucleons with finite density and the phenomenological part of the QCD 
sum rules, represented by some spectral density which was taken to be a delta function plus a continuum, whereas in [4] this spectral density was treated using the vector dominance model that was extended to include the delta-hole polarization initiated by pions produced by the $\rho$ meson decay in nuclear matter. However, in the very recent paper [5] it was shown that QCD sum rules analysis results in small change of the $\rho$-meson mass provided the $\rho \mathrm{N}$ scattering amplitude is properly taken into account. Moreover, some effective models [6] also lead only to marginal changes of the $\rho$-meson mass in the matter.

Unfortunately, chiral symmetry alone cannot predict the actual behavior of the inmedium $\rho$-meson mass. Instead, chiral symmetry dictates the correlators of the chiral partners to be identical in a chirally symmetric world. In our case the vector and axial correlation functions should become equal in the chirally restored phase. Consequently, the vector and axial correlators get mixed when the chiral symmetry is partially restored. Such kind of admixture was found in ref. [0] in the case of finite temperature. In this paper we address the same issue to the system with finite baryon density.

The axial vector correlator includes the contributions from pion and $A_{1}$ meson. The pionic part of the axial correlator can be determined rather well at least in the leading order in density, since pion mass remains close to it vacuum value even for the normal nuclear density and pion decay constant $F_{\pi}$ scales with density like $\bar{q} q$ condensate, in-medium behaviour of which can be determined by model-independent way [8] in the linear in nuclear density approximation. The contribution in the axial correlator which is due to $A_{1}$ meson, causes more problems. Unfortunately, there are neither theoretical calculations nor attemps to determine the in-medium properties of $A_{1}$ experimentally. Therefore, to study the vector axial mixing one needs to assume some in-medium behaviour of the unmixed correlators. One notes that, whereas the effect of the mixing is inevitably present as long as chiral symmetry gets restored, one seems impossible to determine quantitatively the in-medium masses of the chiral partners caused by too large uncertainties already on the level of the unmixed vector and axial correlators. However one can determine, at least qualitatively, the density dependence of the $\rho$ - $A_{1}$ mass difference, since this is a parameter characterizing the 
spontaneous breaking of chiral symmetry.

In-medium behaviour of hadrons can be studied theoretically by calculating the twopoint correlator of the corresponding current. In the particalar case of the $\rho$-meson the correlator is given by

$$
\Pi_{\mu \nu}(p)=i \int d^{4} x e^{i p \cdot x}\left\langle\Psi\left|T\left\{V_{\mu}(x) V_{\nu}(0)\right\}\right| \Psi\right\rangle
$$

where $V_{\mu}$ is the isovector current and the matrix element is taken over the ground states of the system with finite baryon density $\rho$. In the case of the noninteracting nucleons the expression above is identical to that, calculated by Hatsuda et.al [3]. However, when nuclear pions are explicitely included, the direct interaction of those pions with the vector currents may induce the mixing of the vector and axial correlators. Admixture of the opposite parity channel arises when two pions interact with vector currents at points 0 and/or $x$. The piece of the correlator where the vector current interacts directly with the nuclear pion that is emitted and then absorbed by the nuclear matter can be written in the form

$$
\sum_{a, b} \int \frac{d^{3} \mathbf{k}}{2 \omega_{k}} \frac{d^{3} \mathbf{k}^{\prime}}{2 \omega_{k^{\prime}}}\left\langle\Psi\left|a^{a \dagger}(\mathbf{k}) a^{b}\left(\mathbf{k}^{\prime}\right)\right| \Psi\right\rangle i \int d^{4} x e^{i p \cdot x}\left\langle\Psi \pi^{a}(\mathbf{k})\left|T\left\{V_{\mu}(x) V^{\mu}(0)\right\}\right| \Psi \pi^{b}\left(\mathbf{k}^{\prime}\right)\right\rangle,
$$

In the nuclear matter the pions are virtual so one needs to consider all possible time orderings where two pions are either emitted or absorbed by the matter. The corresponding pieces of the correlator can be represented by the expressions similar to Eq.(2) but with two forward - or backward going pions. One has, respectively

$$
\frac{1}{2} \sum_{a, b} \int \frac{d^{3} \mathbf{k}}{2 \omega_{k}} \frac{d^{3} \mathbf{k}^{\prime}}{2 \omega_{k^{\prime}}}\left\langle\Psi\left|a^{a}(\mathbf{k}) a^{b}\left(\mathbf{k}^{\prime}\right)\right| \Psi\right\rangle i \int d^{4} x e^{i p \cdot x}\left\langle\Psi\left|T\left\{V_{\mu}(x) V^{\mu}(0)\right\}\right| \Psi \pi^{a}(\mathbf{k}) \pi^{b}\left(\mathbf{k}^{\prime}\right)\right\rangle,
$$

and

$$
\frac{1}{2} \sum_{a, b} \int \frac{d^{3} \mathbf{k}}{2 \omega_{k}} \frac{d^{3} \mathbf{k}^{\prime}}{2 \omega_{k^{\prime}}}\left\langle\Psi\left|a^{a \dagger}(\mathbf{k}) a^{b \dagger}\left(\mathbf{k}^{\prime}\right)\right| \Psi\right\rangle i \int d^{4} x e^{i p \cdot x}\left\langle\Psi \pi^{a}(\mathbf{k}) \pi^{b}\left(\mathbf{k}^{\prime}\right)\left|T\left\{V_{\mu}(x), V^{\mu}(0)\right\}\right| \Psi\right\rangle
$$

where $\omega_{k}=\sqrt{\mathbf{k}^{2}+m_{\pi}^{2}}$.

We consider the nuclear pions in the chiral limit so that the nuclear wave functions appearing in the momentum and space integrals are the same in the leading chiral order 
implying that in this order pion absorption or/and emission does not change the nuclear wave functions. One notes, that similar approach [9] was used earlier to remove the unwanted pieces forbidden by chiral symmetry in the QCD sum rules for the nucleon mass in matter. In some sence in ref. [9] and in the present paper the same issue, namely the consistency of the QCD sum rules calculations of hadron properties in the nuclear matter, is addressed. In case of $\rho$-meson this amounts to necessity to include into consideration the in-medium behaviour of its chiral partner, $A_{1}$ meson. In other words, any study of the $\rho$-meson mass in matter is not fully consistent with chiral symmetry, unless the in-medium modifications of $A_{1}$ meson is included. Using the soft-pion theorem one has

$$
i\left\langle\Psi \pi^{a}(\mathbf{k})\left|T\left\{V_{\mu}(x), V^{\mu}(0)\right\}\right| \Psi \pi^{b}\left(\mathbf{k}^{\prime}\right)\right\rangle \simeq \frac{-i}{f_{\pi}^{2}}\left\langle\Psi\left|\left[Q_{5}^{a},\left[Q_{5}^{b}, T\left\{V_{\mu}(x), V^{\mu}(0)\right\}\right]\right]\right| \Psi\right\rangle
$$

while the matrix elements in both (3) and (4) also reduce to the same expression. Calculating the double commutator and making use of the usial expansion of the pion field in terms of creation and annihilation operators and commutation relations of current algebra $\left[Q_{5}^{a}, J_{\nu}^{b}\right]=$ $i \epsilon^{a b c} A_{\nu}^{c}$ one can get the following expression for $\Pi_{V}$

$$
\Pi_{V}=\stackrel{\circ}{\Pi}_{V}+2 \xi\left(\stackrel{\circ}{\Pi}_{V}-\stackrel{\circ}{\Pi}_{A}\right)
$$

and similar expression for the axial correlator $\Pi_{A}$

$$
\Pi_{A}=\stackrel{\circ}{\Pi}_{A}+2 \xi\left(\stackrel{\circ}{\Pi}_{A}-\stackrel{\circ}{\Pi}_{V}\right)
$$

Here $\stackrel{\circ}{\Pi}_{V}\left(\stackrel{\circ}{\Pi}_{A}\right)$ is the correlator of the vector (axial) currents calculated in the approximation of the noninteracting nucleons with finite density. We defined

$$
\xi=\frac{\rho \bar{\sigma}_{\pi N}}{f_{\pi}^{2} m_{\pi}^{2}}
$$

and used the relation

$$
\bar{\sigma}_{\pi N}=4 \pi^{3} m_{\pi}^{2}<N\left|\pi^{2}(0)\right| N>
$$

where $\bar{\sigma}_{\pi N} \simeq-25 \mathrm{MeV}$ is the leading nonanalytic term in the $\sigma_{\pi N}$ corresponding to the contribution of the pion mass term to the nucleon mass and $\pi(0)$ is the pion field. From the 
Eqs. (6-7) one can see that the complete mixing arises at $\rho \simeq 3 \rho_{0}$ but this estimate should not be taken too seriously, since both the in-medium condensates and phenomenological spectral anzats get highly model dependent at such densities and reliable conclusion cannot be drawn. Moreover, the soft-pion approximation may no longer be valid near the point of chiral symmetry restoration. It is worth mentioning that, even at the normal nuclear density, where the change of the two-quark condensate can almost entirely be described by the model independent, linear in nuclear density term, only the qualitative conclusions about the inmedium behaviour of axial and vector meson mass difference can be made. This is basically due to unknown behaviour of the correlator of the axial currents in the nuclear medium. We assume that density dependence of $\stackrel{\circ}{\Pi}_{A}$ is the same as that for $\stackrel{\circ}{\Pi}_{V}$. Besides, the higher twists contributions and the factorization approximation, which is clearly too crude to be used in nuclear matter, make the OPE for the vector correlator full of uncertainties too. One notes, that there is no distinction between the longitudinal and transverse directions in nuclear medium and, as such, the corresponding correlators can be related to each other $\Pi_{T}=\omega^{2} \Pi_{L}$ and $3 \omega^{2} \Pi_{L}=-g^{\mu \nu} \Pi_{\mu \nu}$ so only the longitudinal correlation function is really needed. Representing the correlators by the standard expressions containing pole terms plus continuum contributions, starting at some effective thresholds and then applying the Borel transform one can get the following sum rules

$$
F_{\rho}^{2} \exp \left(-M_{\rho}^{2} / M^{2}\right)-\frac{1}{8 \pi^{2}} M^{2} \exp \left(-S_{V} / M^{2}\right)=\stackrel{\circ}{\Pi}_{V}+2 \xi\left(\stackrel{\circ}{\Pi}_{V}-\stackrel{\circ}{\Pi}_{A}\right)
$$

and

$$
F_{A_{1}}^{2} \exp \left(-M_{A_{1}}^{2} / M^{2}\right)+F_{\pi}^{2}-\frac{1}{8 \pi^{2}} M^{2} \exp \left(-S_{A_{1}} / M^{2}\right)=\stackrel{\circ}{\Pi}_{A_{1}}+2 \xi\left(\stackrel{\circ}{\Pi}_{A_{1}}-\stackrel{\circ}{\Pi}_{V}\right)
$$

The unmixed correlators are given by

$$
\begin{gathered}
\stackrel{\circ}{\Pi}_{V}=\stackrel{\circ}{F}_{\rho}^{2} \exp \left(-\stackrel{\circ}{M}_{\rho}^{2} / M^{2}\right)-\frac{1}{8 \pi^{2}} M^{2} \exp \left(-S_{V} / M^{2}\right) \\
\stackrel{\circ}{\Pi}_{A_{1}}=\stackrel{\circ}{F}_{A_{1}}^{2} \exp \left(-\stackrel{\circ}{M}_{A_{1}}^{2} / M^{2}\right)+\stackrel{\circ}{F}_{\pi}^{2}-\frac{1}{8 \pi^{2}} M^{2} \exp \left(-S_{A_{1}} / M^{2}\right)
\end{gathered}
$$


Here $\stackrel{\circ}{F}$ and $\stackrel{\circ}{M}$ is the density dependent residue and mass of the corresponding hadron calculated without the pion corrections. In case of the $\rho$-meson we used the results obtained in Ref. 10] for $\stackrel{\circ}{F}$ and $\stackrel{\circ}{M}$. In principle effective thresholds are also different with and without pionic corrections. However, the numerical effect is small and, therefore, we keep effective thresholds the same. In the practical calculations we used the values $S_{A_{1}}=2.2 \mathrm{GeV}$ and $S_{V}=1.5 \mathrm{GeV}$. As we already mentioned, the in-meidium behaviour of $\stackrel{\circ}{F}_{A_{1}}$ and $\stackrel{\circ}{M}_{A_{1}}$ is completely unknown so we assumed that they scale like the corresponding parameters of the vector spectral density, starting from the values $\stackrel{\circ}{F}_{A_{1}}^{2}=0.021 G e V^{2}$ and $\stackrel{\circ}{M_{A_{1}}}=1.26 \mathrm{GeV}$ at zero density. The density dependence of $\stackrel{\circ}{F}_{\pi}$ can be found from the in-medium analog of the GOR relation $F_{\pi}^{2} M_{\pi}^{2}=2 M_{q}<q \bar{q}>_{\rho}$ making use of the fact that the pion mass remains almost unchanged at least at normal nuclear density and using the values of $<q \bar{q}>_{\rho}$ both with and without pionic corrections obtained by Weise et.al [1]. One notes that at $\rho=\rho_{0}$ the value of $F_{\pi}^{2}$ is quite close to $\stackrel{\circ}{F}_{\pi}^{2}$ since at normal nuclear density meson corrections gives only minor contribution to the quark condensate [11]. In order to obtain the expressions for $M_{A_{1}}$ and $M_{\rho}$ one needs to get rid of the unknown factors $F_{A_{1}}$ and $F_{\rho}$. It can be done by differentiating the sum rules over the variable $-1 / M^{2}$ and dividing the obtained expression by the original sum rules. The corresponding masses $M_{\rho}, M_{A_{1}}$ are shown in Fig.1 as the functions of the nuclear density. One can see from Fig.1 that, although the rho mass still decreases with an increase of the nuclear density, the size of this effect is significantly smaller than in the case of the unmixed correlator, whereas the $A_{1}$ meson mass drops considerably faster resulting in the convergence of the rho and $A_{1}$ masses. It may indicate the tendency toward the partial chiral symmetry restoration. One can also try to use the other model for the phenomenological part of the vector correlator developed in Ref. [5] and leading to the almost unchanged in-medium mass of the rho-meson. As one can see from Fig.2 in this case $M_{\rho}$ grows with density while $M_{A_{1}}$ still decreases, albeit quite slowly. It is worth mentioning that, in spite of the distinctions in the density dependence for the vector and axial meson masses, obtained in different models, the mass difference $M_{\rho}-M_{A_{1}}$ remains approximately 
the same in all cases. However, one needs to stress again that the above results are obtained under the assumption that $\stackrel{\circ}{\Pi}_{V}$ and $\stackrel{\circ}{\Pi}_{A}$ scale identically with density. One could do the complete QCD sum rules calculations for the axial channel but the uncertainties will be at least as large as those for QCD sum rules analysis of the in-medium rho-mass so it seems unlikely that such culculations will significantly reduce the ambiguities in the value of the $A_{1}$ mass in the nuclear matter.

In this paper we have presented the analysis of the pionic corrections in the QCD sum rules for the vector and axial vector mesons in the nuclear matter. It was found that such corrections result in the effect of mixing of the correlators of the opposite parity, calculated in the approximation when the nuclear matter was treated as a medium of the noninteracting nucleons with some finite density. The mass difference between the chiral partners $\rho$ and $A_{1}$ decreases with the growth of the nuclear density indicating the tendency towards the partial restoration of chiral symmetry. However, chiral symmetry alone cannot predict the actual values of the meson masses in the nuclear medium due to uncertainties both in the in-medium quark and gluon condensates (especially in the 4-quark condensate) and in the phenomenological spectral densities. The standard anzats represented by the sum of pole and continuum seems to be unadequate to get reliable density dependence of the in-medium masses. In order to go beyond the normal nuclear density one needs to consider some additional effects. First,the soft-pion approximation may become invalid if the density is high enough so at some point the finite pion momenta should be taken into account. Second, at high densities, when the $\rho-A_{1}$ mass difference may become comparable with the typical energy of the nuclear transitions, the exitation of the residual nucleus should be included so that the effect of mixing of vector and axial vector correlators will be represented by the sum over $p-h$ states with certain parity to maintain the corresponding conservation law. Besides, at high densities the direct interactions of the isovector current with heavy scalar mesons may also give a significant contribution. 


\section{ACKNOWLEDGEMENTS}

The author gratefully acknowledge useful discussions with M.Birse, T.Hatsuda and S.H.Lee.

[1] R.D.Pisarski, Phys. Lett. 110 (1982) 155;

[2] ,G. E. Brown and M. Rho, Phys. Rev. Lett. 66 (1991) 438;

[3] T.Hatsuda, S.H.Lee, Phys. Rev. C46 (1992) 34;

[4] , M.Asakawa, C.M.Ko, Nucl. Phys. A560 (1993) 399;

[5] F.Klingl,W.Weise, hep-ph/9702240;

[6] R.Rapp, J.Wambach, Phys. Lett. B315 (1993) 220; G.Chanfray, P.Schuck, Nucl.Phys. A555 (1993) 329;

[7] M. Dey, V. L. Eletsky and B. L. Ioffe, Phys. Lett. B252 (1990) 620.

[8] E. G. Drukarev and E. M. Levin, Nucl. Phys. A511 (1990) 679; A516 (1990) 715(E).

[9] M.C.Birse and B.V.Krippa, Phys. Lett. 381 (1996) 397;

[10] X.Jin, D. Leinweber, nucl-th/9510064;

[11] R.Brockmann, W.Weise, Phys. Lett. 367 (1996) 40; 


\section{FIGURES CAPTIONS}

Fig.1 Density dependence of the mass (in GeV) of the $A_{1}$ (upper curve) and $\rho$ (lower curve) mesons expressed in the units of the normal nuclear density $\rho_{0}$ when the pole plus continuum model [3,10] is used for $\stackrel{\circ}{\Pi}_{V}$ and $\stackrel{\circ}{\Pi}_{A_{1}}$.

Fig.2 The same as in Fig.1a but with the use of the model for $\stackrel{\circ}{\Pi}_{V}$ and $\stackrel{\circ}{\Pi}_{A_{1}}$ suggested in [5]. 
Fig. 1

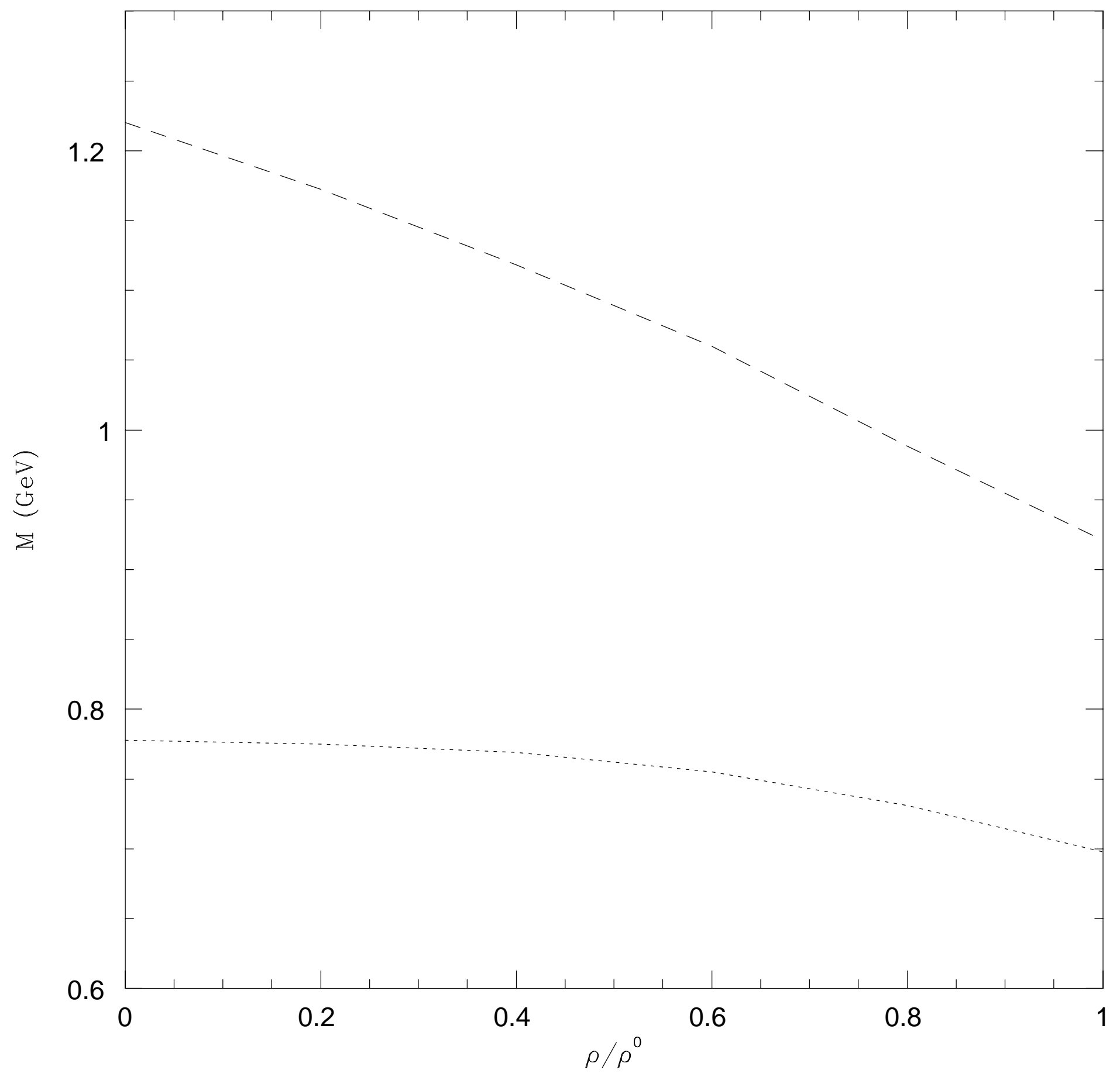


Fig. 2

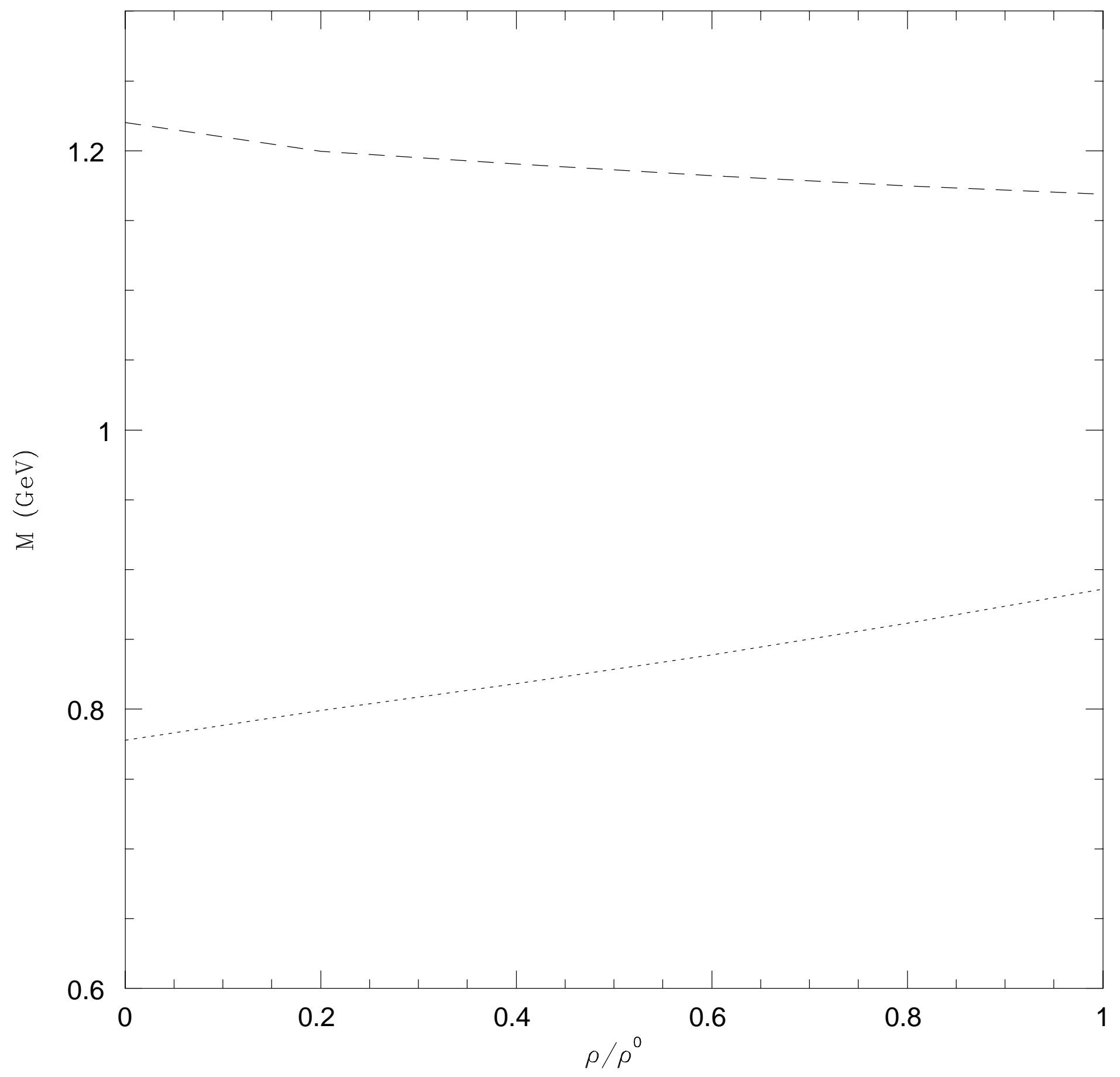

